\title{
Initial Gamma Knife radiosurgery for nonfunctioning pituitary adenomas
}

\author{
Clinical article
}

\author{
Cheng-Chia Lee, M.D., ${ }^{1,3,4}$ Hideyuki Kano, M.D., Ph.D., ${ }^{2}$ Huai-Che Yang, M.D.,, 3 \\ Zhiyuan Xu, M.D., ${ }^{1}$ Chun-Po Yen, M.D., ${ }^{1}$ Wen-Yuh Chung, M.D.,${ }^{3,4}$ \\ David Hung-Chi Pan, M.D., ${ }^{3,4}$ L. Dade Lunsford, M.D., ${ }^{2}$ \\ and Jason P. Sheehan, M.D., Ph.D. ${ }^{1}$
}

${ }^{1}$ Department of Neurological Surgery, University of Virginia Health System, Charlottesville, Virginia; ${ }^{2}$ Department of Neurosurgery, University of Pittsburgh, Pennsylvania; ${ }^{3}$ Department of Neurosurgery, Neurological Institute, Taipei Veterans General Hospital; and ${ }^{4}$ School of Medicine, National Yang-Ming University, Taipei, Taiwan

Object. Nonfunctioning pituitary adenomas (NFAs) are the most common type of pituitary adenoma and, when symptomatic, typically require surgical removal as an initial means of management. Gamma Knife radiosurgery (GKRS) is an alternative therapeutic strategy for patients whose comorbidities substantially increase the risks of resection. In this report, the authors evaluated the efficacy and safety of initial GKRS for NFAs.

Methods. An international group of three academic Gamma Knife centers retrospectively reviewed outcome data in 569 patients with NFAs.

Results. Forty-one patients (7.2\%) underwent GKRS as primary management for their NFAs because of an advanced age, multiple comorbidities, or patient preference. The median age at the time of radiosurgery was 69 years. Thirty-seven percent of the patients had hypopituitarism before GKRS. Patients received a median tumor margin dose of 12 Gy (range 6.2-25.0 Gy) at a median isodose of 50\%. The overall tumor control rate was $92.7 \%$, and the actuarial tumor control rate was $94 \%$ and $85 \%$ at 5 and 10 years postradiosurgery, respectively. Three patients with tumor growth or symptom progression underwent resection at 3,3, and 96 months after GKRS, respectively. New or worsened hypopituitarism developed in 10 patients $(24 \%)$ at a median interval of 37 months after GKRS. One patient suffered new-onset cranial nerve palsy. No other radiosurgical complications were noted. Delayed hypopituitarism was observed more often in patients who had received a tumor margin dose $>18 \mathrm{~Gy}(\mathrm{p}=0.038)$ and a maximum dose $>36$ Gy $(p=0.025)$.

Conclusions. In this study, GKRS resulted in long-term control of NFAs in $85 \%$ of patients at 10 years. This experience suggests that GKRS provides long-term tumor control with an acceptable risk profile. This approach may be especially valuable in older patients, those with multiple comorbidities, and those who have endocrine-inactive tumors without visual compromise due to mass effect of the adenoma.

(http://thejns.org/doi/abs/10.3171/2013.11.JNS131757)

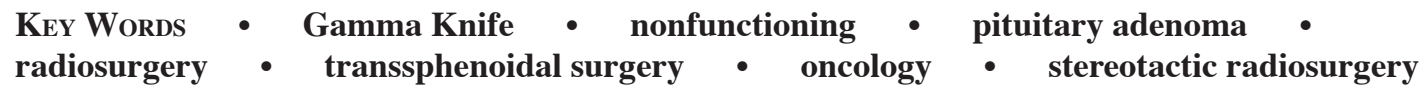

$\mathrm{W}$ HEN intervention is required because of progressive tumor growth or visual compromise, resection is the initial approach for most patients with nonfunctioning pituitary adenomas (NFAs). Such adenomas are not endocrine active, and they comprise

\footnotetext{
Abbreviations used in this paper: $\mathrm{ACTH}=$ adrenocorticotropic hormone; $\mathrm{CN}=$ cranial nerve; $\mathrm{GH}=$ growth hormone; $\mathrm{GKRS}=$ Gamma Knife radiosurgery; NAGKC = North American Gamma Knife Consortium; NFA = nonfunctioning pituitary adenoma; NIS = Nationwide Inpatient Sample; SRS = stereotactic radiosurgery; TSS $=$ transsphenoidal surgery.
}

approximately $30 \%$ of all pituitary adenomas. ${ }^{11,24}$ Unlike secretory pituitary adenomas, NFAs can remain clinically hidden until they become large enough to cause headache, endocrinological abnormalities, or visual field deficits. Gamma Knife radiosurgery (GKRS) is usually considered for patients with recurrent or residual NFA after an initial transsphenoidal resection. Several studies have reported a promising tumor control rate of $80 \%-90 \%$ at 10

This article contains some figures that are displayed in color online but in black-and-white in the print edition. 
years after GKRS and a low risk of visual or endocrine complications. ${ }^{9,26}$ Although the incidence of delayed pituitary dysfunction in one or more hormone axes varies from $30 \%$ to $40 \%$ after GKRS, hormone deficiencies are readily managed with appropriate replacement therapy. Initial resection is not always feasible for NFA patients with significant comorbidities or an advanced age, and some patients prefer a less invasive option if it has satisfactory outcomes. In recent decades, relatively few publications have described the results of primary GKRS for NFA. 2,9,11,20,24,28,30,32 In this study, the outcomes of initial GKRS for NFAs obtained by an international group of three centers with extensive radiosurgical experience were evaluated.

\section{Methods}

\section{Patient Population}

All three medical centers received independent internal review board approval to submit their retrospective clinical outcome analyses of patients with NFAs. Initial or upfront GKRS was defined as GKRS as the primary management modality for patients with NFAs. None of the patients had undergone any prior surgery or fractionated radiation therapy. The medical records of the patients were retrospectively reviewed at each center. A common spreadsheet database with specific variables was established and used by all participating centers. With institutional review board approval, each group reviewed and analyzed pooled and de-identified data and drafted a report.

\section{Study Inclusion Criteria}

For inclusion in the study, all patients had one or more of the following: 1) no history of cancer; 2) contrast-enhanced MRI findings consistent with a pituitary adenoma, as interpreted by the treating clinicians and a radiologist; and 3) a comprehensive endocrinological assessment that demonstrated no evidence of hormonal hypersecretion. All patients also had to have one or more clinical, neuroimaging, and endocrinological follow-up evaluations.

\section{Clinical and Radiographic Evaluations}

Imaging studies consisted of MRI with axial and coronal pre- and postcontrast sequences. Endocrinological studies included serum measurements of growth hormone $(\mathrm{GH})$, insulin-like growth factor-1, adrenocorticotropic hormone $(\mathrm{ACTH})$, cortisol, prolactin, triiodothyronine (T3), thyroxine (T4), free T4, thyrotropin-stimulating hormone, luteinizing hormone, follicle-stimulating hormone, and either estrogen in females or testosterone in males.

After GKRS, all patients underwent clinical and endocrinological evaluations at an average of 6- to 12-month intervals. An attending neurosurgeon and a radiologist reviewed the follow-up neuroimaging studies. Tumor progression was defined as an increase of at least $10 \%$ in tumor volume. Tumor regression was defined as at least a $10 \%$ decrease in tumor volume. A tumor size that was $\pm 10 \%$ of its original volume was defined as stable. ${ }^{27}$ No change or a reduction in tumor volume was defined as tumor control. Pituitary dysfunction related to radiosurgery was defined as a new or worsened endocrine deficiency after GKRS.

\section{Gamma Knife Radiosurgery Technique}

Patients underwent stereotactic frame placement after the application of a local anesthetic supplemented with conscious sedation as needed. Afterward, all patients underwent thin-slice stereotactic MRI with and without intravenous contrast administration. Radiosurgery was performed using either the Leksell Gamma Knife Model 4C or Perfexion unit (Elekta Instrument, Inc.).

\section{Statistical Analysis}

Data are presented as the median or mean and the range for continuous variables and as frequency and percentages for categorical variables. Statistical analyses of categorical variables were performed using the chi-square test, Fisher exact test, and Mantel-Haenszel test for linear association, as appropriate. Statistics of the means were performed using the unpaired Student t-test both with and without equal variance (Levene test), as necessary, and the Wilcoxon rank-sum tests when variables were not normally distributed. A Kaplan-Meier curve was plotted for progression-free survival from the time of GKRS, last follow-up, and failure of treatment if any.

The logistic regression model was used to analyze the prognostic factors of hypopituitarism. Significant variables and interaction expansion covariates were further assessed in both logistic multivariate analyses, as deemed relevant. Probability values $<0.05$ were considered statistically significant. For statistical analysis, we used IBM's SPSS (version 20.0).

\section{Results}

\section{Patient Characteristics}

Forty-one patients who had undergone upfront GKRS between 1988 and 2012 were collected from this multicenter experience of 569 patients with NFAs: 11 patients from the University of Virginia Health System (representing $5.1 \%$ of patients with NFAs treated using GKRS and no prior treatment at the University of Virginia); 15 from the University of Pittsburgh Medical Center (representing 12.0\%); and 15 from Taipei Veterans General Hospital (representing 6.6\%). The median patient age was 69 years, and $51 \%$ of the cohort were women (Table 1). The median tumor volume was $2.3 \mathrm{ml}$ (range $0.2-10.5 \mathrm{ml}$ ). On the initial MRI studies, microadenomas (adenomas $<1$ $\mathrm{cm}$ in diameter) were present in 6 patients $(14.6 \%)$, but the remaining tumors $(85.4 \%)$ were macroadenomas. Thirteen patients (32\%) had NFAs with suprasellar extension, whereas 21 patients $(51 \%)$ had NFAs with some degree of cavernous sinus extension. Fifteen patients (37\%) had pituitary dysfunction in one or more hormonal axes before GKRS. Nine patients had evidence of baseline dysfunction in the thyroid axis, 5 had a deficiency in the cortisol axis, 4 had dysfunction in the gonadotropin axis, and 2 had deficiencies in the GH axis. Five patients had mild elevation of serum prolactin levels that were interpreted as consistent with stalk effect (that is, a serum prolactin level $<150 \mathrm{ng} / \mathrm{ml})$. Nine patients $(22 \%)$ had partial vi- 
TABLE 1: Characteristics in 41 patients who underwent GKRS as primary treatment for NFA*

\begin{tabular}{lc}
\hline \multicolumn{1}{c}{ Characteristic } & Value \\
\hline median age in yrs (range) & $69(30-88)$ \\
sex (F/M) & $21: 20$ \\
median tumor vol in ml (range) & $2.3(0.2-10.5)$ \\
no. of macroadenomas (\%) & $35(85.4)$ \\
no. of patients w/ CS extension (\%) & $21(51.2)$ \\
no. of patients w/ suprasellar extension (\%) & $13(31.7)$ \\
no. of patients w/ pre-GKRS hypopituitarism (\%) & $15(36.6)$ \\
no. of patients w/ pre-GKRS visual deficits (\%) & $9(22.0)$ \\
median imaging FU in mos (range) & $48(7-159)$ \\
median clinical FU in mos (range) & $52(7-159)$ \\
\hline
\end{tabular}

* CS = cavernous sinus; FU = follow-up.

sual field deficits before GKRS, with most cases caused by a primary ocular problem such as macular degeneration, diabetic retinopathy, or cataract. None of the patients had visual deficits related to optic apparatus compression. Twenty patients $(48.8 \%)$ were more than 70 years old, and 30 patients $(73.2 \%)$ had major medical comorbidities that portended high operative risks, including heart failure, chronic obstructive pulmonary disease, poor lung function, stroke, diabetes mellitus, end-stage renal disease, hematological disease, and psychiatric disorders. Eight patients $(19.5 \%)$ were unwilling to undergo more invasive surgical approaches and therefore chose GKRS.

All patients underwent GKRS as the primary or upfront therapy. Prescribed doses (that is, radiosurgical doses to the tumor margin) varied from 6.2 to 25 Gy (median 12 Gy) at a median 50\% isodose level (range 30\%-61\%). The median maximum radiation dose delivered to the optic apparatus was 7.7 Gy (optic chasm), 7.8 Gy (optic nerve), and $8.1 \mathrm{~Gy}$ (optic tract). The maximum radiosurgical dose to the optic apparatus was $<11.2$ Gy (Table 2).

\section{Tumor Control}

The median neuroimaging follow-up was 48 months (range 7-159 months). In all, 18, 13, and 8 patients had $\geq 3, \geq 5$, and $\geq 7$ years of follow-up, respectively. At the last follow-up after GKRS, a decrease in tumor volume occurred in 34 patients $(82.9 \%)$, tumor stability in 4 patients $(9.8 \%)$, and a tumor increase in 3 patients $(7.3 \%)$. Actuarial progression-free survival was $94 \%$ and $85 \%$ at 5 and 10 years postradiosurgery, respectively (Fig. 1). Images from a patient who had an excellent tumor response are featured in Fig. 2.

Of the 3 patients with an increase in tumor size, one had worsened visual field deficits associated with the tumor enlargement. The enlarged adenomas were found on neuroimaging studies in the other 2 patients, who had no clinical symptoms or signs (Table 3 ). The patients with tumor progression received 10,12 , and 12 Gy at the tumor margin to the $30 \%, 50 \%$, and $50 \%$ isodose lines, respectively. There was no statistical difference in the prescription dose between the patients with and without tumor control $(\mathrm{p}>0.05)$.
TABLE 2: Radiosurgical parameters in 41 patients who underwent upfront GKRS for NFA

\begin{tabular}{lcc}
\hline \multicolumn{1}{c}{ Parameter } & Value & Range \\
\hline median tumor margin radiation dose (Gy) & 12.0 & $6.2-25.0$ \\
median maximum radiation dose (Gy) & 26.0 & $17.4-70.0$ \\
median isodose level (\%) & 50 & $30-61$ \\
median treatment vol (ml) & 3.2 & $0.3-11.3$ \\
median optic chiasm radiation exposure (Gy) & 7.7 & $1.2-11.1$ \\
median optic nerve radiation exposure (Gy) & 7.8 & $6.1-11.2$ \\
median optic tract radiation exposure (Gy) & 8.1 & $6.3-11.2$ \\
\hline
\end{tabular}

Neurological Results and Complications

Twenty-one patients (51\%) complained of headaches prior to GKRS, and 3 of them (14\%) had subjective improvement in their headaches at the last follow-up (Table 3). Of the 9 patients with preexisting visual field deficits, $1(11 \%)$ experienced both subjective and objective visual improvement after GKRS. At the last follow-up, 1 patient without tumor enlargement demonstrated new oculomotor and trochlear nerve dysfunction 96 months after radiosurgery. Except for hypopituitarism, radiation-related neurological complications without tumor growth were observed in 1 patient $(2.4 \%)$.

\section{Hypopituitarism}

The median endocrinological follow-up was 52 months (range 7-159 months). None of the 15 patients with pituitary dysfunction before GKRS experienced endocrine improvement as a result of the radiosurgery. Newonset or worsened pituitary deficiencies were detected in $24.4 \%$ of the patients $(n=10$; Table 3$)$. The new or worsened pituitary deficiencies were seen in the following axes: cortisol axis $(n=4)$, gonadotroph axis $(n=4), G H$ axis $(n=3)$, and thyroid axis $(n=2)$. The median time to the development of a new pituitary hormone deficiency was 37 months. In this highly select group of patients, a prescribed tumor margin dose $>18 \mathrm{~Gy}$ and a maximum dose $>36$ Gy were the only 2 prognostic factors for postGKRS hypopituitarism $(\mathrm{p}=0.013$ and 0.011 on univariate analysis, and $\mathrm{p}=0.038$ and 0.025 on multivariate analysis, respectively). Other factors, including age, sex, tumor margin dose, tumor extension, and pre-GKRS hormone status, were not statistically related to hypopituitarism in this series (Table 4).

\section{Additional Management After GKRS}

Three patients had tumor growth after GKRS, and 2 of them underwent resection. The patient with new-onset visual deficits and tumor enlargement underwent transsphenoidal surgery (TSS) 3 months after GKRS. The other patient who had tumor enlargement without clinical symptoms and signs underwent TSS 3 months after GKRS, and the NFA was found to be larger than on the initial MR image with suprasellar extension. The third patient had only mild tumor growth (slightly greater than a $10 \%$ increase in tumor volume) 7 months after GKRS; thereafter, the tumor remained stable 97 months after GKRS. 


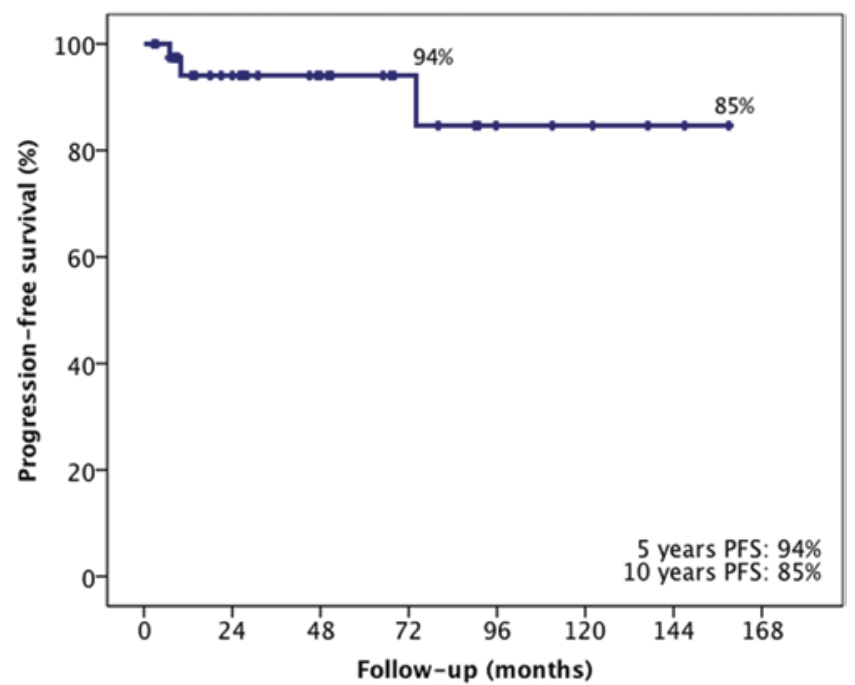

FIG. 1. Kaplan-Meier plot demonstrating progression-free survival (PFS) from the time of GKRS in patients with NFAs treated with upfront GKRS. The numbers of patients having $\geq 3, \geq 5$, and $\geq 7$ years of follow-up were 18,13 , and 8 , respectively. The actuarial progressionfree survival rate was $94 \%$ and $85 \%$ at 5 and 10 years postradiosurgery, respectively.

The patient who demonstrated new-onset cranial nerve $(\mathrm{CN})$ palsy (CNIII and CNIV palsy) at 96 months postradiosurgery had no evidence of tumor enlargement. The initial tumor was larger $(5.5 \mathrm{ml})$ than the median adenoma volume $(2.3 \mathrm{ml})$ treated in this series. Although resection was performed at the onset of the $\mathrm{CN}$ palsies, both palsies remained.

In each of the 3 cases requiring resection, histology showed a typical pattern of null cell adenoma without silent staining characteristics.

\section{Discussion}

Resection remains the first-line option in managing patients with symptomatic NFA. Surgical removal has the advantage of yielding a histological diagnosis and providing information about subtype analysis (for example, silent ACTH subtype). It can also provide rapid relief of mass effect on the neuroendocrine, cerebrovascular, and neuronal structures. Given that many NFAs are macroadenomas, the benefits of relieved mass effect, particularly on the optic apparatus, cannot be understated. If complete resection can be achieved, the procedure can be curative, although delayed recurrences after gross-total resection are frequent and can occur in $24 \%-80 \%$ of cases. ${ }^{2,4,16,17,21}$ The risks of TSS are low, particularly at experienced centers. However, in a recent review of the Nationwide Inpatient Sample (NIS) database, the authors noted that the risks of transsphenoidal resection lead to a $0.7 \%$ rate of inpatient mortality, a 5.6\% rate of neurological deficits, and a $42.1 \%$ overall risk of complications. ${ }^{23}$ Another national report collecting data from 958 neurosurgeons showed that after TSS the complication of pituitary dysfunction would occur in $19.4 \%$ patients and the complication of diabetes insipidus would appear in $17.8 \%$ patients. ${ }^{6}$ In

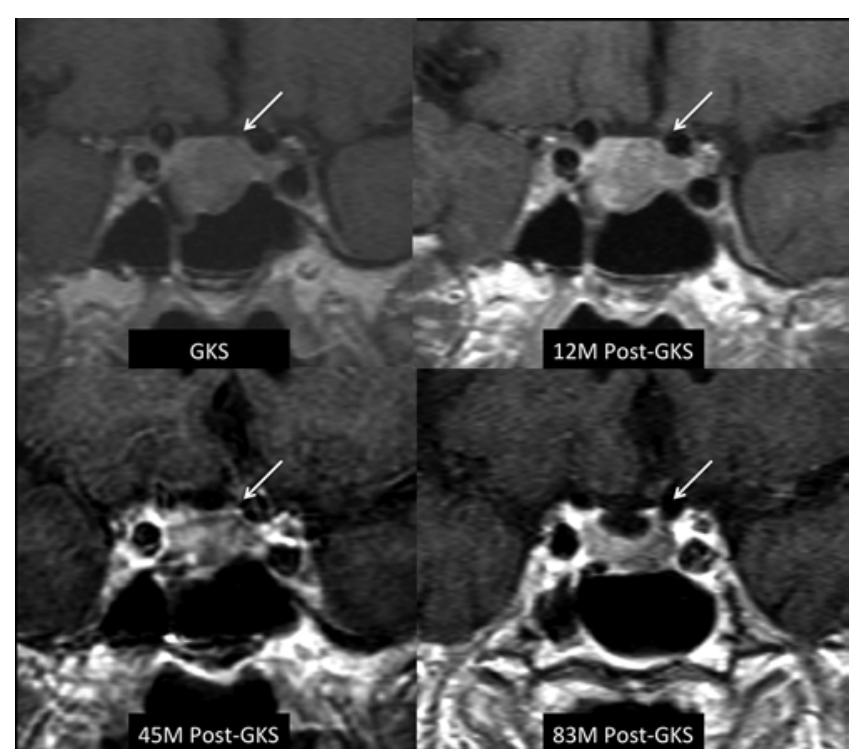

FIG. 2. Images obtained in a 78-year-old woman presenting with headache, mild visual deficits, and blurred vision caused by cataract. An MR image revealed an NFA (arrows) measuring $20 \times 18 \times 18 \mathrm{~mm}$ in size. Neuroendocrine evaluation revealed no deficits. The macroadenoma received a radiosurgical dose of $12 \mathrm{~Gy}$ to the tumor margin. A series of follow-up MR images demonstrate gradual but significant shrinkage. The patient is currently 87 years old, and her hormone status remains normal 101 months after GKRS.

the study of the NIS database, the patients with preoperative comorbidities and those over the age of 64 years were statistically more likely to have an adverse outcome following TSS. ${ }^{23}$ Although a comparison of initial GKRS with transsphenoidal resection seems appropriate, there are patient selection differences that make a comparison between the current study results and the outcomes in the NIS database an imperfect one. In the current study of upfront GKRS for NFA, direct radiosurgical complications were seen in 11 patients (26.8\%), with the majority of the complications (24.4\%) due to hypopituitarism, which is a manageable complication. Age and comorbidities in the current series did not increase the risk of post-GKRS complications.

The use of radiosurgery as initial management for functioning adenomas has been described in previous publications. Castinetti et al. ${ }^{3}$ described 99 patients who underwent radiosurgery for acromegaly, 19 (19\%) of them without prior resection. The authors indicated that these 19 patients had tumors deemed unresectable as a result of "laterosellar extension," a contraindication for open surgery because of comorbidities, or that the patients had refused resection. ${ }^{3}$ No significant difference in endocrine remission was reported in the group with radiosurgery alone (21\%), as compared with the group with radiosurgery after a prior transsphenoidal resection (16\%). ${ }^{3}$ Among 25 patients with Cushing's disease treated with radiosurgery, 4 $(16 \%)$ underwent upfront radiosurgery, and 2 of these patients attained endocrine remission. ${ }^{15}$ Other radiosurgical series of pituitary adenomas have included small groups of patients undergoing radiosurgery without a prior resection. ${ }^{12,18,19,29,30}$ The current report is the first to specifically focus on the outcomes of upfront radiosurgery for NFA. 
Although the natural history of NFAs is not clearly defined, several authors have investigated the issue., ${ }^{5,8,21}$ In an analysis from 2011, the authors studied the natural history of nonfunctioning adenomas based on data from 11 studies having a noncomparative single-cohort design. ${ }^{8}$ The follow-up duration in the study ranged from 3 to 15 years. There was a greater tendency for tumor growth in macroadenomas $(>1 \mathrm{~cm})$ and in solid lesions. In our series, $85 \%$ of the patients had solid macroadenomas (median adenoma volume $2.3 \mathrm{ml}$ ), lesions the recent meta-analysis indicated are more likely to demonstrate growth and resulting problems. ${ }^{8}$ Macroadenomas and solid tumors are more likely to grow per the report by Fernández-Balsells et al. ${ }^{8}$ Thus, treating these adenomas seems more appealing given the natural history of nonfunctioning adenomas. In the current study, 34 tumors $(82.9 \%)$ decreased in size after GKRS. Most nonfunctioning adenomas show no growth or slow growth. The tumor regression after initial GKRS in our study would certainly represent a change from the natural history of nonfunctioning adenomas. Among the patients with a stable tumor, it is possible that tumor stability was induced by the Gamma Knife, but it is also possible that these tumors mirrored the natural history of a slow-growing nonfunctioning adenoma and required a longer follow-up to differentiate radiosurgical success or failure.

Gamma Knife radiosurgery is very low risk for patients with NFAs having a small-to-moderate volume. In a study by the North American Gamma Knife Consortium (NAGKC), an adenoma volume under $5 \mathrm{ml}$ provided the best chance for tumor control and neurological preservation after radiosurgery. ${ }^{26}$ In another study by the University of Pittsburgh group, progression-free survival after radiosurgery was reduced if the tumor volume was larger than $4.5 \mathrm{ml} .{ }^{22}$ In a single-institution study by the University of Virginia, progression-free survival was improved for NFAs under $5 \mathrm{ml.} .^{28}$ According to the literature, resection appears advantageous when the adenoma volume is greater than $5 \mathrm{ml}$. For those patients with a smaller NFA, volume reduction to below $5 \mathrm{ml}$ may provide diminishing returns if radiosurgery is to be performed postoperatively. Thus, upfront radiosurgery would seem more appealing for smaller-volume NFAs.

\section{Defining an NFA}

In the current study, MRI coupled with the absence of hormone hypersecretion was the cornerstone of diagnosing NFAs. Exquisite soft-tissue contrast and an ability to interrogate the pituitary gland and parasellar anatomy with high spatial resolution and without artifacts from surrounding bony structures have made MRI the primary modality for evaluating NFAs. ${ }^{10}$ Nonfunctioning pituitary adenomas, similar to functioning adenomas, exhibit an iso- or hypointense T1 signal on MRI as compared with a normal gland signal, unless intratumoral hemorrhage is present. Most NFAs demonstrate a high T2 signal. Most are round or discoid in morphology. Contour deformity of the pituitary gland and infundibulum may be present. After intravenous gadolinium injection, NFAs are relatively hypo-enhancing or iso-enhancing as compared with a normal pituitary gland. If dynamic imaging is performed,
TABLE 3: Outcomes in 41 patients who underwent upfront GKRS for NFA*

\begin{tabular}{|c|c|c|}
\hline Parameter & No. $(\%)$ & $\begin{array}{l}\text { Months to Occur } \\
\text { (range) } \dagger\end{array}$ \\
\hline \multicolumn{3}{|l|}{ no. of tumor responses } \\
\hline shrinkage & $34(82.9)$ & \\
\hline stability & $4(9.8)$ & \\
\hline enlargement & $3(7.3)$ & \\
\hline \multicolumn{3}{|l|}{ no. of clinical improvements } \\
\hline headache relief & $3(14.3)$ & \\
\hline discontinue hormone replacement & $0(0)$ & \\
\hline visual improvement & $1(11.1)$ & \\
\hline \multicolumn{3}{|l|}{ no. of complications } \\
\hline radiation necrosis & $0(0)$ & - \\
\hline visual deterioration & $1(2.4) \ddagger$ & 3 \\
\hline new CN palsy & $1(2.4)$ & 96 \\
\hline cerebrovascular accident & $0(0)$ & - \\
\hline hypopituitarism & $10(24.4)$ & $37(24.0-73.0)$ \\
\hline $\mathrm{ACTH}$ & 4 & \\
\hline gonadotroph & 4 & \\
\hline $\mathrm{GH}$ & 3 & \\
\hline thyroid & 2 & \\
\hline panhypopituitarism & $0(0)$ & - \\
\hline diabetes insipidus & $0(0)$ & - \\
\hline \multicolumn{3}{|l|}{ no. w/ further management } \\
\hline TSS for tumor resection & 3 & $3,3,96$ \\
\hline radiotherapy or radiosurgery & 0 & - \\
\hline
\end{tabular}

* The actuarial radiation-related complication rate except hypopituitarism was $2.4 \%$.

† Blank spaces indicate that the data were unavailable; $-=$ not applicable.

$\ddagger$ The visual deterioration was present with tumor enlargement.

the differential uptake of contrast between an adenoma and the normal pituitary gland serves as another effective diagnostic tool. ${ }^{1}$

Once MRI is consistent with a pituitary adenoma, the next step is to prove that the adenoma is a nonfunctioning one. By definition, the diagnosis of NFA should include the finding of no over-production of hormones in a comprehensive endocrinology evaluation; however, a low percentage of NFA cells stain for corticotropin, GH, prolactin, or thyrotropin. Such tumors are referred to as a "silent" corticotrophic, somatotrophic, lactotrophic, or thyrotrophic adenoma, respectively. ${ }^{32}$ The diagnosis of silent adenoma would be impossible to exclude from the current series because the patients did not undergo prior resection, although none had any demonstrable hypersecretion pituitary hormone upon comprehensive neuroendocrine testing. Of the 3 patients who ultimately underwent a resection after GKRS, all had tumors consistent with NFAs without silent staining features. Thus, while histology remains the gold standard, diagnosis of an NFA by neuroimaging, clinical, and endocrine features alone seems a reasonable approach. 
TABLE 4: Prognostic factors of hypopituitarism after GKRS

\begin{tabular}{|c|c|c|c|c|}
\hline \multirow[b]{2}{*}{ Parameter } & \multicolumn{2}{|c|}{ №. (\%) } & \multirow[b]{2}{*}{ Univariate Analysis } & \multirow[b]{2}{*}{ Multivariate Analysis } \\
\hline & Not Present & Present & & \\
\hline no. of patients & 31 & 10 & & \\
\hline mean age in yrs & $67.8 \pm 13.0^{*}$ & $61.7 \pm 23.2^{*}$ & 0.296 & - \\
\hline female sex & $16(51.6)$ & $5(50.0)$ & 0.929 & - \\
\hline tumor margin dose $>18 \mathrm{~Gy}$ & $1(3.2 \%)$ & $4(40)$ & 0.013 & 0.038 \\
\hline maximum dose $>36 \mathrm{~Gy}$ & $3(9.7 \%)$ & $5(50)$ & 0.011 & 0.025 \\
\hline $\begin{array}{l}\text { mean tumor vol in } \mathrm{ml} \\
\text { tumor extension }\end{array}$ & $3.6 \pm 2.6^{*}$ & $4.7 \pm 3.2^{*}$ & 0.290 & - \\
\hline CS invasion & $11(35.5)$ & $2(20.0)$ & 0.360 & - \\
\hline suprasellar expansion & $16(51.6)$ & $5(50.0)$ & 0.929 & - \\
\hline
\end{tabular}

*Values are presented as the means \pm standard deviation. $-=$ not applicable.

\section{Other Advantages to Initial GKRS Management of NFAs}

Invasion of the adenoma into the cavernous sinus has also been shown to be a challenge for complete resection. Such an invasion can often be determined on preoperative MRI. Knosp et al. ${ }^{14}$ developed a grading system to predict cavernous sinus invasion on the basis of preoperative MRI. For adenomas located between or beyond the intercarotid line and the lateral tangent (that is, Grade 2, 3, or 4), cavernous sinus invasion of the adenoma is likely. ${ }^{14}$ Cavernous sinus invasion has been shown to be a risk factor for recurrent or residual tumor after resection of an NFA..$^{20}$ In addition, pituitary adenomas exhibiting cavernous sinus invasion have been shown to have a higher growth rate, as measured by $\mathrm{Ki} 67$ analysis of adenoma specimens..$^{13}$ Thus, targeting the cavernous sinus component of the pituitary adenoma would seem important and of therapeutic value in any surgical approach. Radiosurgery, unlike microsurgery, can be extended into the cavernous sinus with relative ease. Stereotactic radiosurgery (SRS) is often used to encompass adenomas in the sella as well as those invading the cavernous sinus in one approach and to convey a low risk of cranial neuropathy. In a recent series from the North American Gamma Knife Consortium (NAGKC), the risk of cranial neuropathy after GKRS for an NFA was quite low even in patients with cavernous sinus invasion. ${ }^{26}$ As such, upfront radiosurgery for adenomas residing primarily or exclusively in the cavernous sinus may be an appealing approach.

In addition, the efficacy of SRS for NFAs has proven durable. In the current study, 5-year actuarial tumor control was $94 \%$. According to the study by the NAGKC, overall tumor control was achieved in $93.4 \%$ of patients at the last follow-up. ${ }^{26}$ Actuarial tumor control was $98 \%$, $95 \%, 91 \%$, and $85 \%$ at $3,5,8$, and 10 years postradiosurgery, respectively. In other words, we can expect that $85 \%$ of patients will not need any further management for their NFA within 10 years, with only minimal complications. If the patient has a smaller tumor volume and no suprasellar extension, tumor control can be even better. ${ }^{26}$

\section{Complications Associated With Upfront GKRS}

In terms of postradiosurgery complications in the NAGKC study, new or worsened hypopituitarism af- ter radiosurgery was noted in $21.0 \%$ of the patients who had NFAs, with thyroid and corticotroph deficiencies reported as the most common postradiosurgical endocrinopathies. ${ }^{26}$ In the present study, the result was similar, with $24.4 \%$ of patients experiencing hypopituitarism at a median of 37 months post-GKRS. A higher radiosurgical dose was related to an increased risk of hypopituitarism after GKRS. Fortunately, a lower tumor margin dose for NFAs, as compared with the dose for functioning adenomas, seems effective for radiosurgery ${ }^{25}$ this lower effective dose for NFAs may translate to a lower risk of delayed hypopituitarism. Even though the incidence of this side effect of SRS seems to be higher than those for microsurgery and TSS, the hypopituitarism is not difficult to manage. Most patients can be treated with the physiological replacement of a diminished hormone. Moreover, from prior publications we have gained a better understanding of the causes of postradiosurgical hypopituitarism and the ways to avoid it. For example, patients receiving a lower dose to the normal gland, pituitary stalk, and hypothalamus are less likely to develop hypopituitarism., ${ }^{71}$ Definite longitudinal neuroendocrine follow-up is needed to detect and correct delayed hypopituitarism if it occurs after radiosurgery. However, long-term and serious complications associated with initial GKRS seem rare.

\section{Role of Initial GKRS in the Management of NFAs}

In summary, resection remains the preferred initial treatment for most patients with NFAs requiring intervention. However, upfront radiosurgery for NFA may be indicated in select clinical settings such as in older patients (70 or more years); in patients with multiple comorbidities in whom an operation would involve a high risk; in patients with clear neuroimaging and neuro-endocrine evidence of an NFA, no mass effect on the optic apparatus, and progressive tumor on neuroimaging follow-up; or in patients who wish to avoid resection. When physicians are faced with such situations, upfront SRS treatment may provide a reasonable alternative for controlling NFAs.

\section{Study Limitations}

The main limitation of this report is its retrospective nature and relatively small patient population. Because of 
its retrospective nature, we were not blinded to the decision of GKRS treatment or to the post-SRS imaging evaluation, both of which are limitations. The small patient population undoubtedly limited the statistical power of some of the analyses. And although the three contributing centers represent academic tertiary care centers and each has Gamma Knife experience of more than 20 years, the diverse referral pathways may have introduced a selection bias and could represent patient cohorts not typical of community or regional hospitals, especially the two US centers in which patients came from local, regional, and international referring physicians. Finally, there were differences in the radiosurgical technique between the centers.

\section{Conclusions}

For selected patients with NFAs, upfront GKRS afforded a high rate of tumor control and preserved neurological function. Hypopituitarism occurred in $24.4 \%$ patients, and other complications (that is, $\mathrm{CN}$ palsy) were observed in $2.4 \%$ of patients. In the future, the selective application of upfront SRS for radiographically and endocrinologically identified NFAs may be reasonable. Further evaluation seems warranted to better define the role of SRS, as compared with the standard approach of resection, for small- to moderate-volume NFAs without the need for immediate relief of mass effect.

\section{Disclosure}

Dr. Lunsford is a consultant and shareholder in Elekta AB. No funds from Elekta were used to complete this study. The authors report no conflict of interest concerning the materials or methods used in this study or the findings specified in this paper.

Author contributions to the study and manuscript preparation include the following. Conception and design: Sheehan, Lee, Xu. Acquisition of data: Lee, Kano, Yang, Xu. Analysis and interpretation of data: Sheehan, Lee, Kano, Yang, Xu, Chung. Drafting the article: Lee. Critically revising the article: all authors. Reviewed submitted version of manuscript: all authors. Approved the final version of the manuscript on behalf of all authors: Sheehan. Statistical analysis: Sheehan, Lee, Kano, Xu, Lunsford. Administrative/technical/material support: Sheehan, Chung, Pan, Lunsford. Study supervision: Sheehan, Chung, Pan, Lunsford.

\section{References}

1. Bartynski WS, Lin L: Dynamic and conventional spin-echo MR of pituitary microlesions. AJNR Am J Neuroradiol 18: 965-972, 1997

2. Brochier S, Galland F, Kujas M, Parker F, Gaillard S, Raftopoulos $\mathrm{C}$, et al: Factors predicting relapse of nonfunctioning pituitary macroadenomas after neurosurgery: a study of 142 patients. Eur J Endocrinol 163:193-200, 2010

3. Castinetti F, Taieb D, Kuhn JM, Chanson P, Tamura M, Jaquet $\mathrm{P}$, et al: Outcome of gamma knife radiosurgery in 82 patients with acromegaly: correlation with initial hypersecretion. J Clin Endocrinol Metab 90:4483-4488, 2005

4. Chandler WF, Schteingart DE, Lloyd RV, McKeever PE, Ibarra-Perez G: Surgical treatment of Cushing's disease. J Neurosurg 66:204-212, 1987

5. Chen Y, Wang CD, Su ZP, Chen YX, Cai L, Zhuge QC, et al: Natural history of postoperative nonfunctioning pituitary ad- enomas: a systematic review and meta-analysis. Neuroendocrinology 96:333-342, 2012

6. Ciric I, Ragin A, Baumgartner C, Pierce D: Complications of transsphenoidal surgery: results of a national survey, review of the literature, and personal experience. Neurosurgery 40: 225-237, 1997

7. Feigl GC, Pistracher K, Berghold A, Mokry M: Pituitary insufficiency as a side effect after radiosurgery for pituitary adenomas: the role of the hypothalamus. Clinical article. J Neurosurg 113 Suppl:153-159, 2010

8. Fernández-Balsells MM, Murad MH, Barwise A, GallegosOrozco JF, Paul A, Lane MA, et al: Natural history of nonfunctioning pituitary adenomas and incidentalomas: a systematic review and metaanalysis. J Clin Endocrinol Metab 96: 905-912, 2011.

9. Gopalan R, Schlesinger D, Vance ML, Er EL, Sheehan J: Longterm outcomes following Gamma Knife radiosurgery for patients with a nonfunctioning pituitary adenoma. Neurosurgery [epub ahead of print], 2011

10. Hess CP, Dillon WP: Imaging the pituitary and parasellar region. Neurosurg Clin N Am 23:529-542, 2012

11. Höybye C, Rähn T: Adjuvant Gamma Knife radiosurgery in non-functioning pituitary adenomas; low risk of long-term complications in selected patients. Pituitary 12:211-216, 2009

12. Jagannathan J, Sheehan JP, Pouratian N, Laws ER, Steiner L, Vance ML: Gamma Knife surgery for Cushing's disease. J Neurosurg 106:980-987, 2007

13. Knosp E, Kitz K, Steiner E, Matula C: Pituitary adenomas with parasellar invasion. Acta Neurochir Suppl (Wien) 53:65-71, 1991

14. Knosp E, Steiner E, Kitz K, Matula C: Pituitary adenomas with invasion of the cavernous sinus space: a magnetic resonance imaging classification compared with surgical findings. Neurosurgery 33:610-618, 1993

15. Kobayashi T, Kida Y, Mori Y: Gamma knife radiosurgery in the treatment of Cushing disease: long-term results. J Neurosurg 97 (5 Suppl):422-428, 2002

16. Laws ER, Ebersold MJ, Piepgras DG: The results of transsphenoidal surgery in specific clinical entities, in Laws ER, Randall RV, Kern EB, et al (eds): Management of Pituitary Adenomas and Related Lesions With Emphasis on Transsphenoidal Microsurgery. New York: Appleton-Century-Crofts, 1982, pp 277-305

17. Laws ER Jr, Vance ML: Radiosurgery for pituitary tumors and craniopharyngiomas. Neurosurg Clin N Am 10:327-336, 1999

18. Lim YL, Leem W, Kim TS, Rhee BA, Kim GK: Four years' experiences in the treatment of pituitary adenomas with gamma knife radiosurgery. Stereotact Funct Neurosurg 70 (Suppl 1):95-109, 1998

19. Liu X, Kano H, Kondziolka D, Park KJ, Iyer A, Shin S, et al: Gamma knife stereotactic radiosurgery for drug resistant or intolerant invasive prolactinomas. Pituitary 16:68-75, 2013

20. Losa M, Mortini P, Barzaghi R, Ribotto P, Terreni MR, Marzoli SB, et al: Early results of surgery in patients with nonfunctioning pituitary adenoma and analysis of the risk of tumor recurrence. J Neurosurg 108:525-532, 2008

21. O'Sullivan EP, Woods C, Glynn N, Behan LA, Crowley R, O'Kelly P, et al: The natural history of surgically treated but radiotherapy-naïve nonfunctioning pituitary adenomas. Clin Endocrinol (Oxf) 71:709-714, 2009

22. Park KJ, Kano H, Parry PV, Niranjan A, Flickinger JC, Lunsford LD, et al: Long-term outcomes after gamma knife stereotactic radiosurgery for nonfunctional pituitary adenomas. Neurosurgery 69:1188-1199, 2011

23. Patil CG, Lad SP, Harsh GR, Laws ER Jr, Boakye M: National trends, complications, and outcomes following transsphenoidal surgery for Cushing's disease from 1993 to 2002. Neurosurg Focus 23(3):E7, 2007 
24. Pollock BE, Cochran J, Natt N, Brown PD, Erickson D, Link $\mathrm{MJ}$, et al: Gamma knife radiosurgery for patients with nonfunctioning pituitary adenomas: results from a 15-year experience. Int J Radiat Oncol Biol Phys 70:1325-1329, 2008

25. Sheehan JP, Niranjan A, Sheehan JM, Jane JA Jr, Laws ER, Kondziolka D, et al: Stereotactic radiosurgery for pituitary adenomas: an intermediate review of its safety, efficacy, and role in the neurosurgical treatment armamentarium. J Neurosurg 102:678-691, 2005

26. Sheehan JP, Starke RM, Mathieu D, Young B, Sneed PK, Chiang VL, et al: Gamma Knife radiosurgery for the management of nonfunctioning pituitary adenomas: a multicenter study. Clinical article. J Neurosurg 119:446-456, 2013

27. Snell JW, Sheehan J, Stroila M, Steiner L: Assessment of imaging studies used with radiosurgery: a volumetric algorithm and an estimation of its error. Technical note. J Neurosurg 104:157-162, 2006

28. Starke RM, Williams BJ, Jane JA Jr, Sheehan JP: Gamma Knife surgery for patients with nonfunctioning pituitary macroadenomas: predictors of tumor control, neurological deficits, and hypopituitarism. Clinical article. J Neurosurg 117:129135,2012

29. Thorén M, Rähn T, Guo WY, Werner S: Stereotactic radiosur- gery with the cobalt- 60 gamma unit in the treatment of growth hormone-producing pituitary tumors. Neurosurgery 29:663668,1991

30. Wowra B, Stummer W: Efficacy of gamma knife radiosurgery for nonfunctioning pituitary adenomas: a quantitative follow up with magnetic resonance imaging-based volumetric analysis. J Neurosurg 97 (5 Suppl):429-432, 2002

31. Xu Z, Lee Vance M, Schlesinger D, Sheehan JP: Hypopituitarism after stereotactic radiosurgery for pituitary adenomas. Neurosurgery 72:630-637, 2013

32. Yamada S, Kovacs K, Horvath E, Aiba T: Morphological study of clinically nonsecreting pituitary adenomas in patients under 40 years of age. J Neurosurg 75:902-905, 1991

Manuscript submitted August 14, 2013.

Accepted November 18, 2013.

Please include this information when citing this paper: published online January 3, 2014; DOI: 10.3171/2013.11.JNS131757.

Address correspondence to: Jason P. Sheehan, M.D., Ph.D., Departments of Radiation Oncology, Neurological Surgery, and Neuroscience, University of Virginia Health System, P.O. Box 800212, Charlottesville, VA 22908. email: jsheehan@virginia.edu. 